\title{
Meta
}

Journal des traducteurs

Translators' Journal

\section{Le nouveau langage de la médecine : une affaire de socioterminologie}

\section{Christian Balliu}

Volume 50, numéro 4, décembre 2005

Pour une traductologie proactive - Actes

For a Proactive Translatology - Proceedings

Por una traductología proactiva - Actas

URI : https://id.erudit.org/iderudit/019909ar

DOI : https://doi.org/10.7202/019909ar

Aller au sommaire du numéro

Éditeur(s)

Les Presses de l'Université de Montréal

ISSN

0026-0452 (imprimé)

1492-1421 (numérique)

Découvrir la revue

Citer cet article

Balliu, C. (2005). Le nouveau langage de la médecine : une affaire de socioterminologie. Meta, 50(4). https://doi.org/10.7202/019909ar
Résumé de l'article

La médecine est une discipline plurimillénaire profondément ancrée dans l'histoire. Il n'est donc pas étonnant que le langage de la médecine - et par conséquent sa terminologie - ait toujours été influencé par ses utilisateurs. Le traducteur médical doit prendre conscience que le sociolecte médical, loin de reposer sur une terminologie rigoureuse et objective, est soumis à des variations tant synchroniques que diachroniques. 


\title{
Le nouveau langage de la médecine : une affaire de socioterminologie
}

\author{
CHRISTIAN BALLIU \\ ISTI - Haute École de Bruxelles, Bruxelles, Belgique \\ ISIT, Paris, France \\ cballiu@heb.be
}

La maladie est un rapport erroné au monde.

Françoise Dolto

\begin{abstract}
RÉSUMÉ
La médecine est une discipline plurimillénaire profondément ancrée dans l'histoire. Il n'est donc pas étonnant que le langage de la médecine - et par conséquent sa terminologie - ait toujours été influencé par ses utilisateurs. Le traducteur médical doit prendre conscience que le sociolecte médical, loin de reposer sur une terminologie rigoureuse et objective, est soumis à des variations tant synchroniques que diachroniques.
\end{abstract}

\begin{abstract}
An age-old science, medicine has had to follow in the steps of history for millenniums. Little wonder, then, if medical language - and, hence, terminology - has always been influenced, over the years, by its successive users. The medical translator is bound to realize that the medical sociolect, far from feeding on an exact and objective terminology, is in fact prey to synchronic as well as diachronic instability.
\end{abstract}

MOTS-CLÉS/KEYWORDS

traduction médicale, sociolecte, terminologie, connotation, subjectivité

La médecine est une discipline profondément enracinée dans l'histoire. Son caractère diachronique se matérialise aussi bien dans l'évolution de son savoir et de ses dénominations que dans un figement terminologique qui assure la pérennité des notions à travers le temps.

Il existe par conséquent un mouvement de flux et de reflux entre les découvertes médicales et la « philosophie » qui sous-tend l'exercice de la médecine et la conception du monde qu'elle met en œuvre. Toute médecine naît dans un environnement qui la conditionne et qui, à son tour, est infléchi par les nouvelles découvertes. Il suffit pour s'en convaincre de retracer l'évolution des hôpitaux depuis le Moyen Âge, que ce soit en Europe ou sous la dynastie abbasside dans l'Orient médiéval.

La transition, déjà ancienne, entre une médecine rituelle, destinée à refléter le dessein des dieux et donc extérieure à toute volonté objective, et une médecine où le thérapeute recherche la compréhension des phénomènes pathologiques pour devenir l'acteur de la guérison, ou du moins de la rémission, pose les jalons d'une véritable science médicale, justiciable d'un enseignement facultaire et d'une recherche scientifique qui ne sauraient davantage être « de droit divin ».

Cette inversion de la perspective, qui traduit un changement profond dans les mentalités, devait obligatoirement s'accompagner d'une révolution des dénominations. Vouloir sonder les notions, les comprendre, les appréhender, c'est aussi appliquer ses propres grilles de lecture à la séméiologie. Au fond, quand on y réfléchit quelque peu, la médecine est une science vouée à l'échec, dans la mesure où ses interventions, aussi brillantes soient-elles aujourd'hui sur le plan technique, aboutissent inéluctablement, tôt ou tard, au décès du patient. Une course contre le temps 
perdue d'avance, dont la vanité fut mise en exergue pour la première fois par Xavier Bichat à l'aube du XIX ${ }^{\mathrm{e}}$ siècle :

\section{C'est parce que l'homme est mortel qu'il est malade et non pas parce qu'il est malade qu'il meurt. ${ }^{1}$}

Cet aphorisme a imposé une vision programmée de la vie humaine, dont la fin serait un aboutissement naturel, inéluctable, qui à lui seul conditionnerait la morbidité et, dans sa foulée, la létalité. C'est une prescience de l'immunologie, quelque soixante-dix ans avant Pasteur et l'épopée des vaccinations. L'organisme devient dès lors le complice, pour ne pas dire l'artisan, de sa propre destruction à l'aide des forces qui l'avaient jusque là maintenu en vie. D'ailleurs, l'apoptose ${ }^{2}$, découverte plus tard précisément par l'immunologie cellulaire, traduit la mort biologique prédéterminée et inéluctable du patient. Voilà, le mot « patient » a été prononcé. Ce mot - ou s'agirait-il d'un terme ? - recèle au moins deux notions : la souffrance et le sujet pensant, dont le participe présent évoque la participation active à la pathologie et éventuellement à la thérapie. Le concept d'agent morbide cède donc le pas à un vitalisme systémique ${ }^{3}$.

Les notions et la terminologie du domaine recèlent des choix théoriques dans lesquels l'intuition joue un rôle essentiel. L'allopathie en vigueur aujourd' hui se fonde sur l'aphorisme latin « contraria contrariis curantur », alors que l'homéopathie de Hahnemann bâtit son concept sur l'aphorisme inverse « similia similibus curantur », ce qui inaugure la loi de similitude. On se souviendra aussi de la «théorie des signatures » de Paracelse qui recherche un principe logique, presque sacralisé, derrière l'incohérence apparente de l'univers ; selon cette théorie, si le saule pousse au bord des marais, c'est qu'il contient une substance capable de lutter contre les fièvres palustres et les rhumatismes, dans lesquels l'humidité joue un rôle étiologique patent. L'aspirine, aujourd'hui encore le médicament le plus vendu dans le monde, est le nom donné à l'acide acétylsalicylique (du latin salix : saule).

Par conséquent, même si cela peut paraître curieux en première analyse, l'objectivité qui est à la base de toute science doit nécessairement s'adosser en l'espèce à une subjectivité inébranlable qui est la caution de l'intuition, de la découverte, du génie. Relire la biographie de certains grands patrons est très éclairant à cet égard. Pour le dire autrement, la plupart du temps en médecine, on ne trouve que ce que l'on cherche. Mais il ne faut pas se méprendre : l'intuition des grands ne nait pas in abstracto, elle s'appuie sur une connaissance approfondie du domaine et sur une expérience au long cours. Comment une science qui s'occupe des arcanes physiologiques, mais aussi psychologiques, d'un sujet pourrait-elle ne pas être, en partie du moins, marquée au sceau de la subjectivité ? Comment un médecin pourrait-il investiguer le corps d'un patient en faisant abstraction de ses propres grilles de lecture ? Comment l'anamnèse pourrait-elle faire l'impasse sur le discours?

Les mots trahissent cet état d'esprit. Ils donnent aux termes leur sens en les emplissant de vie. En traduction médicale, être à l'affût des sentiments, des sensations, c'est être à l'écoute du sens. Si la terminologie fait inconsciemment appel à la langue répertoire, les mots ressortissent à la langue système, en ce sens qu'ils organisent l'univers physiologique selon des critères holistiques. Foucault a raison : "Une grammaire de signes s'est substituée à une botanique de symptômes. " ${ }^{4}$

On devine déjà les implications possibles pour la littérature médicale - l'usage du mot « littérature » est révélateur -, et ses traducteurs. La question fondamentale est la suivante : le discours médical cautionne-t-il le caractère scientifique de la discipline par une terminologie, à savoir un lien indéfectible, logique et précis entre une notion et une dénomination, ou ce discours fait-il aussi la part belle aux mots, qui mettent en jeu un lien elliptique, métonymique, voire métaphorique entre la notion et sa matérialisation linguistique ? Dans le prolongement de cette réflexion, il conviendra de s'interroger sur l'action des terminologues qui s'emploient toujours davantage à élargir les contextes au-delà des expressions figées, de la phraséologie ou du microdomaine. Dans leur esprit, le discours l'emporte graduellement sur la langue.

La terminologie a été hantée ces dernières années par le fantôme de l'émiettement. Le foisonnement des notions, la multiplication des dénominations et la complexité croissante des connaissances ont poussé les terminologues à subdiviser les domaines en sous-domaines dans un 
premier temps, en microdomaines ensuite. Une évolution somme toute logique en regard de l'hyperspécialisation croissante, notamment en médecine. Cette hyperspécialisation a aussi une rançon : la dépersonnalisation de la science médicale au profit d'un savoir extérieur au sujet, dont le médecin serait le seul dépositaire en vertu d'une compétence intrinsèque, laquelle ne saurait en aucun cas être partagée avec le patient.

Effectivement, dans la formation reçue ou dans l'exercice de sa profession, le traducteur médical est sensibilisé au premier abord à la terminologie si particulière au discours médical, d'apparence mystérieuse et impénétrable, comme si elle recelait des savoirs insondables par le commun des mortels. Une langue cabalistique en quelque sorte, qui paraît renouer avec la pensée magique, préexistentielle, à la seule différence, remarquable, que le savoir n'est plus transcendantal mais incarné dans la personne, presque immatérielle et inaccessible, du praticien.

À raisonner de la sorte, on pourrait être enclin à penser que le discours médical se résume à la maitrise d'une terminologie reposant sur des acquis notionnels bien particuliers, laquelle cautionnerait la véracité scientifique. La traduction serait dès lors à la fois complexe et aisée. Complexe, dans la mesure où la pratique médicale, justiciable de nombreuses années d'études universitaires, ne saurait être dominée dans son ensemble par un spécialiste des sciences humaines, a fortiori lorsque l'on sait que la médecine s'émiette de plus en plus en disciplines indépendantes qui témoignent de la richesse et de la spécialisation croissantes du domaine. Aisée, parce que le travail du traducteur se résumerait à la recherche de termes univoques, dénotés, dont le sens serait sans surprise puisque la polysémie et l'ambiguïté en seraient évacuées.

Un texte d'Henri Mondor, ce merveilleux chirurgien de l'abdomen du XX $\mathrm{XX}^{\mathrm{e}}$ siècle, illustre la vanité de cette croyance et montre l'immixtion de la connotation dans une description séméiologique :

Une douleur atroce et vive diffusée avec traînée à gauche [...]. Peu de vomissements [...], une diarrhée fétide, choléiforme. Une température peu élevée : $38^{\circ}$ [...]. Mais un pouls à 140, faible, fuyant. Le faciès est plombé, tiré, gris, hébété, anxieux...5

Le texte, pourtant extrait d'un ouvrage de médecine spécialisé, acquiert une dimension presque littéraire, rappelant certaines descriptions médicales que l'on retrouve chez Hervé Bazin. La dichotomie classique entre textes littéraires et textes scientifiques doit, à mon sens, être revue à la lumière de tels exemples et ils sont légion.

Cette connotation de la littérature médicale, souvent à des fins de simplification et de clarification, peut à mon sens se remarquer à partir de la deuxième moitié du XIX ${ }^{\mathrm{e}}$ siècle, qui vit le triomphe de la médecine d'observation, dite « médecine clinique », où le patient, après des siècles d'obscurantisme imposés par la Faculté, se retrouve au centre de l'attention, grâce aux techniques conjuguées d'auscultation, de palpation et de percussion. L'usage de l'ouie, la réhabilitation du regard et la manipulation des membres mirent à l'honneur un vocabulaire courant pour décrire cette gestuelle. Ne parle-t-on pas aujourd'hui encore d'un « geste» lorsque l'on propose une intervention à un patient? De grands médecins comme René Laennec et Claude Bernard ne sont bien entendu pas étrangers au phénomène et ce n'est pas là leur moindre apport à la médecine de leur temps.

A propos de Laennec, l'histoire du mot stéthoscope ne manque pas d'intérêt. Étymologiquement, je serais tenté de dire «terminologiquement », le mot désigne un instrument destiné à visionner la poitrine. Un stéthoscope ne sert bien entendu pas à cela mais à une multitude d'autres choses. La biunivocité se recherche désespérément dans l'étymologie et conduira immanquablement à conclure que le mot ne reflète ni sa fonction ni son usage. D'où vient donc ce mot?

Laennec appliquait la méthode anatomoclinique qui s'appuie sur l'observation minutieuse de l'organisme du patient. Si le mal provient du thorax, il convient donc d'utiliser l'auscultation « immédiate » en plaçant son oreille sur la peau pour écouter les battements du cœur et le souffle des poumons. Mais lorsqu'en 1816, Laennec, d'une timidité extrême, reçoit une malade 
apparemment cardiaque, il estime que « le sexe de la malade interdit ce genre d'examen ». La volonté scientifique est tout de même la plus forte :

Je vins à me rappeler un phénomène d'acoustique fort connu : si l'on applique l'oreille à l'extrémité d'une poutre, on entend très distinctement un coup d'épingle donné à l'autre bout. J'imaginai que l'on pourrait peut-être tirer parti de cette propriété. Je pris un cahier de papier, j'en formai un rouleau dont j'appliquai une extrémité sur la région précordiale et, posant l'oreille à l'autre bout, je fus aussi surpris que satisfait d'entendre les battements du cœur d'une manière beaucoup plus nette et plus distincte que je ne l'avais jamais fait par application ${ }^{6}$ directe de l'oreille.

L'auscultation médiate était née. Le rouleau de papier devint rapidement un cylindre en bois que Laennec appela d'abord pectoriloque - terme moins critiquable - et dénomma enfin stéthoscope ${ }^{8}$, sans doute en souvenir de l'épisode de 1816. La relation entre la fonction et la dénomination est en l'espèce ponctuelle, accidentelle, en aucun cas scientifique.

En négligeant la dimension diachronique de la science médicale dans un souci d'actualisation souvent mal compris, nombre de terminologues ont privilégié dans leur analyse des domaines de spécialité le linguistique au détriment du discursif. En fait, l'unicité du discours médical n'est qu'un leurre, justement parce que la médecine a pris conscience, dans la seconde moitié du XX $\mathrm{XX}^{\mathrm{e}}$ siècle, qu'elle est aussi une science du logos. À ce propos, le succès croissant de la psychiatrie, qui était naguère encore la branche la plus délaissée et la plus critiquée de la médecine, a joué un rôle crucial. La psychiatrie avait cependant proposé il y a un siècle déjà une nouvelle vision synthétique de l'homme malade. Celle-ci avait échoué dans un premier temps, notamment parce que la communauté scientifique déniait au patient une part active dans la morbidité et la description séméiologique.

Dans le même temps, la médecine contemporaine, hyperspécialisée, vouée à ventiler l'exploration du corps humain en une multitude de sous-domaines, luttait pied à pied contre toute intrusion du logos. La fragmentation organique ou physiologique favorisait la dépersonnalisation de la relation médecin-patient pour se fondre en une relation spécialiste-organe. La conjonction de la dépersonnalisation et de l'hyperspécialisation poussa les observateurs extérieurs, dont les linguistes, à penser que le discours médical ne pouvait être qu'impersonnel, déniant à l'individu toute existence autonome, psychologique, en dehors d'un schéma hiérarchique délibérément réducteur. L'émiettement de la médecine en spécialités autonomes ventilait par le fait même le patient en entités organiques indépendantes et déniait une approche systémique.

Aujourd'hui encore, malgré l'arrivée sur le devant de la scène de l'immunologie, qui restaure la communication entre les domaines de spécialité par une vision horizontale, décloisonnante, le principe fondamental de la médecine contemporaine demeure que le corps et ses fonctions s'expriment en termes physico-chimiques et physiologiques et que l'idéal, pour un médecin, doit être de devenir un ingénieur chimiste, physicien, voire électronicien de cette centrale énergétique qu'est le corps humain. La prolifération et la complexité des techniques médicales, qu'elles soient diagnostiques ou thérapeutiques, ont limité en grande mesure le contact du médecin avec son malade, en réduisant celui-ci à une juxtaposition d'organes. En d'autres termes, le malade tend insidieusement à s'effacer devant la maladie.

Au plan réflexif, la dialectique entre la médecine clinique, toute puissante jusqu 'à l'aube de la Seconde Guerre mondiale, et la médecine de laboratoire fait passer d'une époque où l'intuition et l'observation avaient la primauté, à une ère où la puissance diagnostique des dosages est telle que la clinique n'est plus que le prélude au biologique. Ainsi, la distribution voulue des spécialités engendre ipso facto une structuration lexicale qui se matérialise dans les principes d'inclusion et de hiérarchie si commodes au plan méthodologique.

C'est précisément cette approche méthodologique, fondée sur une classification en tiroirs, qui a été privilégiée par la terminologie pour éluder les vases communicants polysémiques. Il s'agit ni plus ni moins que de circonscrire l'usage d'un terme ou d'un syntagme à un domaine particulier dont il ne pourrait s'échapper en gardant le même sens. Le sens équivaudrait dès lors à une signification prise dans l'étau d'un domaine de spécialité au monologue singulier. 
Ce faisant, on évacue sans autre forme de procès l'approche transversale, interdisciplinaire, qui établit précisément la relation entre plusieurs symptômes ou entre un symptôme, matérialisation d'une pathologie, et son étiologie, qui en est souvent éloignée, du moins en apparence. L'écoute du patient, son anamnèse, en dit parfois plus long - c'est le cas de le dire - sur l'origine de la souffrance que des examens très techniques, mais aléatoires. Le médecin doit être à l'écoute du patient comme celui-ci est à l'écoute de son corps. Le symptôme est souvent polysémique, sa mise en contexte en éclaire le sens profond, physiologique ou psychologique. Il en va de même du texte médical. La monosémie d'apparat cache quelquefois des sens plus profonds, imperceptibles si on ne lit pas sous la surface des signes linguistiques qui sont, eux aussi, la simple matérialisation d'enjeux sémantiques et sémiologiques plus enfouis.

L'exil forcé - au sens où on force un destin - de la polysémie s'est traduit par le recours aux termes en lieu et place des mots. On se rend bien compte que la terminologie paie de la sorte un lourd tribut à la linguistique dont elle est, somme toute, une émanation. La sécheresse d'un diagnostic qui est proféré sans discussion possible, ne permet pas au patient de se dérober, de « jouer sur les mots ». Nous voilà donc au cœur de ce qui semblerait être le nouveau langage de la médecine, qui fractionne le savoir et le réservoir terminologique pour raisonner en termes

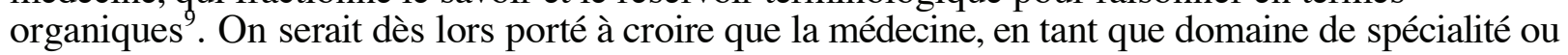
constellation de sous-domaines, se caractérise par un recours systématique aux termes, dénotés par essence, au détriment des mots, des métaphores ou des métonymies qui ne garantiraient ni l'exactitude ni la rigueur de la discipline.

Si l'on devait ainsi accréditer la thèse d'une terminologie scientifique, monoréférentielle et univoque, la distinction entre approches synchronique et diachronique n'aurait aucun sens. Les termes seraient les garants intemporels d'un lien notionnel qui traverse les époques sans dommages, dans la mesure où la constante dénominative serait insensible aux outrages du temps. Ce serait bannir toute évolution du savoir médical et lui contester son statut scientifique.

Ce balisage des connaissances, si commode au plan articulatoire, fait l'impasse sur la vie des termes qui, comme les mots, sont marqués par l'usage. Pour consolider les savoirs dans le temps, il sera bon d'avoir sous la main quelques grands classiques comme le Harrison ou le Garnier Delamare. Ce sont des livres de terrain ${ }^{10}$ qui s'inscrivent de manière résolue dans l'histoire de la médecine. La rapidité de changement du discours médical, l'évolution de sa terminologie, la caducité des nouvelles dénominations rendent plus indispensables encore ces ouvrages de référence, qui donnent une assise diachronique à la discipline. À côté des néologismes, qui actualisent le discours et reflètent l'évolution des techniques depuis la Seconde Guerre mondiale, le lecteur averti retrouvera aussi des mots et des concepts plus anciens, délaissés aujourd'hui, mais qu'il aura côtoyés quelques décennies plus tôt, lors de ses études de médecine par exemple. Il arrivera aussi au médecin expérimenté de recevoir dans son cabinet un patient d'une soixantaine d'années, dont le traitement au long cours aura été entamé jadis, sur la base d'examens, d'explorations et de traitements dont il n'a plus la moindre idée.

En d'autres mots, la terminologie médicale dépend étroitement de l'environnement social qui la voit naître. Elle relève des modes de raisonnement qui induisent les évolutions techniques plutôt qu'ils ne les suivent. L'hyperspécialisation actuelle fait que même l'interniste peut être désarmé devant un patient qui lui parle d'un résultat ou lui cite un nom propre dont il n'a jamais entendu parler. Être spécialisé, c'est aussi méconnaître des pans entiers du savoir médical. C'est là toute la difficulté de rédiger des dictionnaires et des bases de données terminologiques qui conjuguent harmonieusement actualisation du domaine et vision épistémologique large.

Comme je l'ai indiqué, sous l'influence conjointe de l'immunologie et de la psychiatrie, la médecine raisonne de plus en plus de manière systémique, considérant l'individu dans son intégralité, non seulement physiologique, mais aussi animale ${ }^{11}$ ou psychologique. Les nouvelles conquêtes de la biologie moléculaire et le développement de l'épidémiologie ont joué leur rôle dans cette approche.

La psychiatrie a bénéficié en retour de cette nouvelle conception, puisque, en tant qu'étude de la personnalité morbide, elle est devenue désormais la base d'une synthèse inédite dans la pratique médicale. Le domaine de la psychiatrie englobe aujourd'hui non seulement les névroses et les psychoses, mais aussi tous les troubles fonctionnels ou lésionnels, symptomatiques d'un 
trouble de la personnalité humaine ou de son harmonie psychosomatique. Il est en effet peu de maladies, si « organiques » qu'elles soient, où l'influence de la personnalité ne se manifeste pas. Les chirurgiens et les anesthésistes n'ont-ils pas appris à se méfier du futur opéré anxieux, déprimé ou défaitiste? La maladie est effectivement un rapport erroné au monde.

Sous l'influence de l'immunologie et de la psychiatrie, la médecine contemporaine atteint une dimension métaphysique, celle de la constitution du soi, différent des autres soi qui forment le non-soi. L'immunologie devient par conséquent la « science du soi et du non-soi », selon la formule de l'Australien Frank Burnet ${ }^{12}$, prix Nobel de médecine en 1960. L'immunologie crée une théorie du langage des organes, tout comme la psychiatrie impute aux lacunes d'expression et de communication de ses patients leur symptomatologie clinique ${ }^{13}$. De la sorte, nombre d'affections n'ont plus de pathogenèse, mais une psychogenèse ${ }^{14}$ qui témoigne du rôle étiologique actif de l'individu dans la naissance de la maladie. En outre, les maladies auto-immunes (psoriasis, sclérose en plaques, diabète insulinodépendant,...) montrent les limites du soi et l'incertitude d'une identité ontologique clairement définie. Elles remettent aussi en selle les incertitudes sur les causes des maladies et sur l'organisation du système immunitaire dans son ensemble : les pathologies ne sont pas toujours provoquées par des agents infectieux. La maladie se développe en effet au cœur même du cogito et transpire sur ses sentiments.

Dès lors, le discours médical dans son ensemble - car l'immunologie traverse horizontalement la structure physiologique - devient métaphorique et recèle, derrière un style en apparence impersonnel, un combat tout personnel contre la maladie. On parlera ainsi de batterie de tests, de mécanismes de défense, d'arsenal thérapeutique, de cohortes de patients ou encore de stratégie antivirale. Ces tours justiciables d'un lexique militaire renouent avec le sujet pensant qui se mobilise (encore un terme militaire) pour lutter contre l'antigène. Ce discours devient connoté, affectif.

Le problème crucial de la terminologie, médicale en l'occurrence, n'est pas seulement celui du rapport difficile, erratique parfois, entre notion et dénomination. Le lien n'est pas direct, immédiat, transparent ; il est sinueux et emprunte des chemins de traverse. À côté du lésionnel, que l'on croit ingénument plus limité, la terminologie devra aussi prendre en compte le fonctionnel, dont elle n'a souvent pas conscience ou du moins pas pris la mesure. Pour le dire autrement, le ciment est tout aussi important que les briques dans l'appareil de construction et, s'il est moins apparent, il en constitue néanmoins le liant. C'est ainsi que j'ai pu écrire que ce sont les mots qui viennent au secours des termes et qu'un texte bien documenté n'est pas forcément un texte bien traduit ${ }^{15}$.

Il est vrai que certains auteurs souhaiteraient bannir du domaine des maladies psychosomatiques les troubles purement fonctionnels. Ces troubles, même s'ils font souffrir le malade, restent souvent banals et deviennent de plus en plus fréquents. Le « ce n'est rien..., c'est nerveux » peut mener à sous-estimer une chronicité qui ouvre la porte aux maladies psychosomatiques réelles : la gastrite devient ulcère, la fluctuation tensionnelle se mue en hypertension artérielle, etc.

La limite entre trouble fonctionnel et maladie est, dans un certain nombre de cas, assez incertaine. Les stérilités par anovulation d'origine psychique révèlent non seulement une inhibition fonctionnelle, mais peuvent aussi traduire une personnalité gravement en conflit avec elle-même. Alors, trouble fonctionnel ou maladie ? La terminologie éprouve ce type de difficultés classificatoires. Le fonctionnel est en mouvement, il est insaisissable et se laisse difficilement réduire à une appellation ou à une définition nettement circonscrite. Il n'y a derrière le fonctionnel qu'une réalité virtuelle, imaginée mais non imaginaire, dont seule la somatisation pose l'existence. La terminologie ne peut malheureusement s'accommoder du « fantasmatique » ni des fonctions symboliques pourtant si importantes dans la description des signes cliniques.

J'en arrive de cette manière au sociolecte médical, lequel véhicule cette « théorie du langage des organes » que j'évoquais plus haut, mais aussi une théorie du langage des fonctions, dynamique et qui résiste aux tentatives d'hiérarchisation et d'inclusion. Le vocabulaire et les modes d'expression de la médecine sont professionnels, c'est-à-dire qu'ils ne sont pas nécessairement savants. Cela explique également que ce vocabulaire se renouvelle sans cesse ou, mieux, qu'il est renouvelé par la communauté scientifique au fil des découvertes ou des modes. Ainsi le mot gorge $e^{16}$ désigne plusieurs territoires anatomiques et c'est le contexte qui en déterminera le sens in situ. De 
même, l'abandon de l'interprétation hippocratique des maladies fait que l'on recherchera en vain dans le Garnier Delamare des termes tels que ablepsie, acampsie ou acognosie. Le terme abstersion y a fait sa réapparition après avoir été délaissé lors de nombreuses éditions, sans doute à cause de la montée des médicaments abstergents.

Le renouvellement du vocabulaire est tout aussi intéressant. La phtisie du XIX ${ }^{\mathrm{e}}$ siècle est devenue la tuberculose, l'anaphylaxie est devenue l'allergie, raison pour laquelle la confusion entre les deux entrées est entretenue. Dans le domaine psychologique, le terme relaxation, doublet savant de relâchement, s'appliquait auparavant à la paroi abdominale atteinte de hernie. Aujourd'hui, le terme est cantonné à son usage psychologique.

Enfin, la concurrence synonymique est fréquente et montre le volet connotatif de la terminologie médicale. Pour donner un exemple, le syndrome des jambes sans repos, dont la dénomination remonte à Ekbom en 1945, a pour synonymes impatiences, mérasthénie agitante, paresthésie agitante nocturne des membres inférieurs et syndrome d'Ekbom.

Ce nouveau langage de la médecine, souvent latent, impose une nouvelle vision scientifique de l'homme à la première personne, dont la prise en compte dans la traduction est indispensable pour représenter le sociolecte à l'honneur aujourd'hui dans la communauté médicale. La quête des enjeux enfouis sous la surface linguistique déplace l'attention du traducteur vers l'auteur du texte et vers son destinataire, et lui confère un statut dynamique. Souvent la communication médicale vise des créneaux, des crédits de recherche, des débouchés ; elle est parfois plus orientée vers un positionnement dans la communauté scientifique que par la seule volonté de faire progresser la discipline. L'éponymie médicale est une illustration frappante de ce phénomène : que l'on pense à la maladie de Parkinson, au bacille de Hansen ou au sarcome de Kaposi ${ }^{17}$.

La soif de notoriété, le statut universitaire, la reconnaissance des pairs, autant d'alibis qui poussent le spécialiste à se revendiquer de son propre mouvement, à chercher tantôt un père spirituel, tantôt des disciples, afin de créer une école de pensée ou du moins y appartenir. Une originalité de pensée doit être véhiculée par un discours spécifique, qui apporte « quelque chose de nouveau » par rapport à ce qui existait. On omettra ainsi volontiers de citer des sources étrangères dont on s'inspire ou encore des travaux analogues menés sous d'autres cieux, afin de passer pour un précurseur.

La médecine, c'est aussi cela : une lutte d'influences et de pouvoir, un enjeu financier gigantesque. À cet égard, la terminologie joue un rôle capital, car elle symbolise et labellise la notion dans la société ; elle ne peut être que sociolinguistique. C'est plutôt le mot associé à une découverte ou à une avancée qui marque les esprits que le progrès en lui-même. Pour s'en convaincre, il suffit de se rappeler la lutte qui entoura l'émergence de l'anesthésie ${ }^{18}$ en 1846 . Si l'histoire reconnaît aujourd'hui à Wells la priorité, à Jackson l'idée et à Morton l'application, il ne faut pas oublier que les trois protagonistes se disputèrent jusqu'au bout la paternité de l'invention, avec des conséquences dramatiques. Morton emballe son cheval et se tue dans le buggy qui l'emmène à New York voir Jackson ; Wells se suicide en se sectionnant l'artère fémorale, non sans avoir inhalé au préalable des vapeurs anesthésiques. Une ironie de l'histoire.

Tout cela sera invisible pour l'œil d'un lecteur non averti, mais le traducteur médical a pour mission de faire émerger le propos caché et d'éventer la ruse implicite. Les termes ne donnent pas nécessairement la clé du sens ; ils fonctionnent parfois comme un écran qui occulte le message profond du texte. En présence d'un texte médical, le traducteur se doit d'être un médecin des âmes.

\section{NOTES}

1. Bichat X. (1803). Lettre à Pinel, Paris (éditée un an après sa mort).

2. C'est la mort cellulaire programmée, face à certaines pathologies sévères.

3. Il est utile de rappeler ici que Magendie, le père de la physiopathologie, était tout à fait opposé au vitalisme de son époque.

4. Foucault M. (1963). Naissance de la clinique, Paris : Presses Universitaires de France.

5. Mondor H. (1965). Diagnostics urgents - abdomen, Paris : Masson, 9e éd., p. 78. 
6. La troisième occurrence du mot appliquer en trois phrases à peine est symptomatique de la méthode anatomoclinique.

7. Laennec R. T. H. (1819). Traité de l'auscultation médiate et des maladies des poumons et du cour, Paris : Brosson \& Chaudé. On y remarquera le tribut payé par Laennec au Corpus Hippocratique.

8. La Faculté reconnut les mérites du médecin breton en donnant son nom à l'hôpital de Paris qui abritait les incurables. C'était d'ailleurs la première fois qu'on donnait le nom d'un médecin à un hôpital.

9. Voir aussi : Balliu Chr. (2000). «Le traducteur médical et le nouveau langage de la médecine », Hieronymus, revue de l'ASTTI (Association Suisse des Traducteurs, Terminologues et Interprètes), n 4, p. 18-23.

10. Le Dictionnaire Garnier Delamare a vu le jour en 1900 et en est à sa $27^{\mathrm{e}}$ édition. Il est né en fait à l'hôpital Broussais où, en 1897, deux internes, Marcel Garnier et Valery Delamare, ont eu l'idée de rédiger « un petit lexique, à l'usage des étudiants ».

11. Du latin anima, qui signifie âme. Il convient de signaler que la pensée médiévale, si décriée, reliait déjà la science médicale à des disciplines annexes comme l'astrologie, préfigurant ainsi le dualisme cartésien sans renouer avec la protomédecine magique préhippocratique, divinatoire et incantatoire.

12. Parmi les textes fondateurs de l'immunologie moderne, on consultera : Burnet F. M. (1969). Cellular Immunology, Self and Notself, Cambridge: Cambridge University Press.

13. Cela ne signifie pas que le symptôme en question ait obligatoirement une fonction symbolique comme c'est le cas dans l'hystérie.

14. Voir notamment Alexander F. (1952). La médecine psychosomatique (1 $1^{\mathrm{e}}$ éd. 1934), trad. française, Paris : Payot. 15. Balliu Chr. (2004). «La traduction médicale, ou quand les mots viennent au secours des termes », Traduire au $X X I^{e}$ siècle : tendances et perspectives, Actes du Colloque international sur la traduction, Université Aristote de Thessalonique, Faculté des Lettres, p. 103-109.

16. Chose curieuse, l'entrée gorge n'est pas reprise dans la dernière édition du Garnier Delamare.

17. Ce médecin viennois, Moritz Kohn de son vrai nom, décrivit en 1872 le sarcome qui porte son nom et que le sida a remis sous les feux de la rampe.

18. Si le dentiste Morton invente l'anesthésie moderne en 1846 en dépit de fortes oppositions, l'Antidotaire de Nicolas, médecin de l'école de Salerne au $\mathrm{X}^{\mathrm{e}}$ siècle, donnait déjà une recette pour soulager les douleurs du patient. Ce recueil d'ordonnances fournit notamment la description d'une éponge soporifique, imbibée d'un savant mélange d'opium, de jusquiame, de jus de mûre et de laitue, de mandragore et de lierre, que l'on place sous le nez du malade pour l'endormir ou du moins l'apaiser avant l'intervention. Pour d'autres informations à ce sujet, on lira : Balliu Chr. (2000). « La traduction médicale au $\mathrm{XVI}^{\mathrm{e}}$ siècle : une maladie de traducteurs », Nostradamus, traducteur traduit, Actes du colloque international de traductologie, Bruxelles : éd. du Hazard, p. 13-33.

\section{RÉFÉRENCES}

Alexander, F. (1952) : La médecine psychosomatique (1 ${ }^{\mathrm{e}}$ éd. 1934), trad. française, Paris, Payot. BALliU, Chr. (2000) : «Le traducteur médical et le nouveau langage de la médecine », Hieronymus, revue de l'ASTTI (Association Suisse des Traducteurs, Terminologues et Interprètes) 4, p. 18-23.

BALLIU, Chr. (2000) : «La traduction médicale au XVI ${ }^{\mathrm{e}}$ siècle : une maladie de traducteurs », Nostradamus, traducteur traduit, Actes du colloque international de traductologie, Bruxelles, éd. du Hazard, p. 13-33.

BALLIU, Chr. (2004) : « La traduction médicale, ou quand les mots viennent au secours des termes », Traduire au $X X I^{e}$ siècle : tendances et perspectives, Actes du Colloque international sur la traduction, Université Aristote de Thessalonique, Faculté des Lettres, p. 103-109.

Bichat, X. (1803) : Lettre à Pinel, Paris.

BORDET, J. (1920) : Traité de l'immunité dans les maladies infectieuses, Paris, Masson.

Burnet, F. M. (1969): Cellular Immunology, Self and Notself, Cambridge, Cambridge University Press. Foucault, M. (1963) : Naissance de la clinique, Paris : Presses Universitaires de France.

GARnier, M. et V. Delamare (2002) : Dictionnaire des termes de Médecine (27 édition), Paris, Maloine. HAMburger, J. (1982) : Introduction au langage de la médecine, Paris, Flammarion.

HARrison, T. R. (1988) : Principes de Médecine Interne (4 édition), trad. française, Paris, Flammarion.

HIRSCHFELD, L. (1928) : Konstitutionsserologie und Blutgruppensforschung, Berlin, Springer. 
LAEnNeC, R. T. H. (1819) : Traité de l'auscultation médiate et des maladies des poumons et du cœur, Paris, Brosson et Chaudé.

Mondor, H. (1965) : Diagnostics urgents - abdomen, Paris, Masson, $9^{\mathrm{e}}$ éd.

Moulin, A. M. (1991) : Le dernier langage de la médecine. Histoire de l'immunologie de Pasteur au Sida, Paris, Presses Universitaires de France.

Wells, M. G. (1924) : The Chemical Aspects of Immunity, New York, Chemical Catalog Company. 